\title{
Association of diabetes mellitus with trigger finger
}

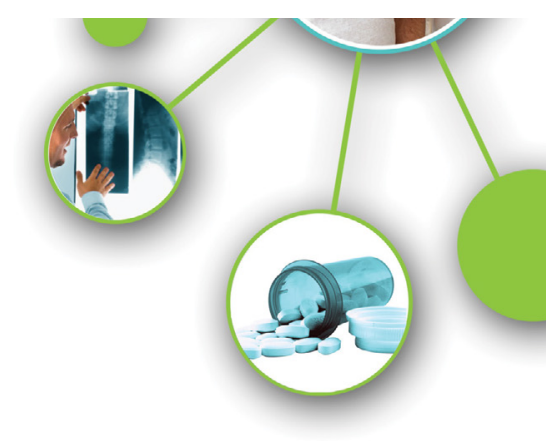

\begin{abstract}
Introduction: High blood sugar in diabetes mellitus affects all system in our body. Hyperglycemia results in many musculoskeletal manifestations involving bones, joints and per articular structures e.g. trigger finger, carpal tunnel syndrome, frozen shoulder, charcot's joint, etc. Trigger finger is may be associated with some risk factors such as diabetes mellitus, rheumatoid arthritis, occupational factors, etc. All the fingers can be affected but more common in thumb, ring and middle finger.
\end{abstract}

Materials and Methods: This study was performed on 103 trigger finger patients attending at OPD of 2 hospitals of Dhaka during the period of July 2017 to June 2019. This is an observational study to see the association of diabetes mellitus with trigger finger.

Results: Out of 103 trigger finger patients 58 (56.31\%) patients had diabetes mellitus. An almost equal percentage of male and female patients with trigger finger had diabetes mellitus. Other risk factors of trigger finger were occupation $(15.53 \%)$, rheumatoid arthritis (2.83\%) and idiopathic (25.24\%). In this observational study, diabetes mellitus was significantly associated with trigger finger.

\section{Keywords: trigger finger, diabetes mellitus, connective tissue, musculoskeletal manifestation}

\section{Introduction}

High blood sugar in diabetes mellitus affects all system in our body [1]. Hyperglycemia causes glycosylation of proteins and less soluble collagen accumulates in the connective tissue. It affects extracellular matrix structure and impairs cell viability $[2,3]$. As a result, there are pathologic alterations in connective tissue, which damage blood vessels and nerves [4]. These ultimately results many musculoskeletal manifestations involving bones, joints and periarticular structures e.g. frozen shoulder, carpal tunnel syndrome, trigger finger, charcot's joint etc. [5-8]. These diseases are very common but often under-recognized and can contribute to significant physical disability and impair quality of life. The actual cause of trigger finger is not known, but it may be associated with some risk factors such as diabetes mellitus, rheumatoid arthritis, occupational factors etc. [9]. When a flexor tendon of finger trapped due to a hypertrophied retinacular sheath at the metacarpal head, it causes triggering and on forced extension it causes a snap. All the fingers can be affected but more common in thumb, ring and middle finger [10]. The aim of the study is to estimate the prevalence of diabetes mellitus in trigger fingers.

\section{Materials and Methods}

\section{Study population}

This study was performed on 103 trigger finger patients attending at OPD of 2 hospitals of Dhaka, National Institute of Traumatology and Orthopedic Rehabilitation (NITOR) and Kurmitola General Hospital (KGH) during the period of July'2017 to June'2019. This is an observational study to see the association of diabetes mellitus with trigger finger. All the patients were underwent history, clinical examination and laboratory examination.

\section{- Study variables}

Study variables were FBS, PPBS, and HbA1C. We also include the following: age, gender, rheumatoid arthritis, and occupation.

\section{Statistical analysis}

The analyses were done in SPSS 22.
Dibakar Sarkar ${ }^{1}$, Taufiq Morshed ${ }^{2}$ and Md. Ashraful Hoque ${ }^{3 *}$

${ }^{1}$ Department of Orthopaedic Surgery, National Institute of Traumatology and Orthopaedic Rehabilitation, Bangladesh

${ }^{2}$ Department of Orthopaedic Surgery, Kurmitola General Hospital, Bangladesh

${ }^{3}$ Centre for Medical Biotechnology, MIS, DGHS, Bangladesh

${ }^{*}$ Author for correspondence: ashraf.djmc03@gmail.com 
Frequencies (expressed as percentage) were used to describe the data.

\section{Results}

Total 103 cases of trigger finger were included in this study attended at OPD of different hospitals in Dhaka. Forty-four patients $(42.72 \%)$ were male and rest $(57.28 \%)$ was female. Mean age of selected patients were 52.09 \pm 10.90 years TABLE 1 .

Out of 103 trigger finger patients, 58 (56.31\%) patients had diabetes mellitus. Among the diabetic trigger finger patients most of the patients (33) are female rest (25) are male patient. About $56 \%$ female trigger finger

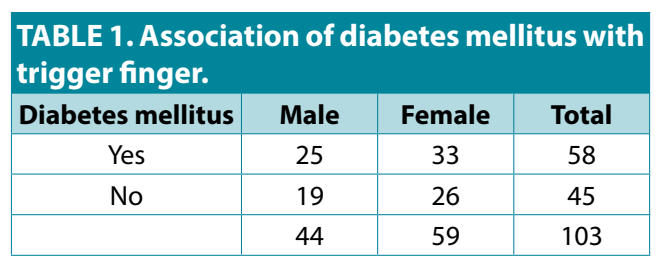

\section{TABLE 2. Other causes of trigger finger.}

\begin{tabular}{|c|c|c|}
\hline Cause & No. of patients & Percentage \\
\hline Occupation & 16 & $15.53 \%$ \\
\hline Rheumatoid arthritis & 3 & $2.83 \%$ \\
\hline Idiopathic & 26 & $25.24 \%$ \\
\hline & 45 & $43.69 \%$ \\
\hline
\end{tabular}

patients are diabetic whereas $59 \%$ males are diabetic TABLE 2.

Trigger finger occurred due to the patient's occupation in $16(15.53 \%)$ patients. Rheumatoid arthritis as a cause of trigger finger occupied a small portion only in three $(2.83 \%)$ patients. The risk factor could not be identified in a significant portion of patients (25.24\%).

\section{Discussion}

In an attempt to find diabetes mellitus as a risk factor of trigger finger we included total of 103 patients attended at OPD in 2 years of study. More than fifty-six percent trigger finger patients were diabetic. An almost equal percentage of male and female patients with trigger finger had diabetes mellitus. Other risk factors of trigger finger were occupation $(15.53 \%)$, rheumatoid arthritis $(2.83 \%)$ and idiopathic (25.24\%). In this observational study, diabetes mellitus was significantly associated with trigger finger.

\section{Conclusion}

There are many risk factors associated with trigger finger. In our observation more than half $(56.31 \%)$ of the patients with trigger finger were diabetic. So, it can be concluded that diabetes mellitus is a significant risk factor for trigger finger. 


\section{References}

Hamdi A, Mousli H, Qari T, et al. Prevalence of trigger finger and carpal tunnel syndrome among diabetic patients and its relationship to He-Moglobin A1C. Indo Amer J Pharma Sci. 5, 16276-16281 (2018).

Crispin J, Alcocer V. Rheumatic manifestations of diabetes mellitus. Am J Med. 14, 753-757 (2003).

Deshmukh DP, AkarteAG. Musculoskeletal manifestations in type 2 diabetes mellitus. Int J Resh Med Sci. 5, 398-402 (2017).
Kamath SU, Ranranatha Y. Musculoskeletal and joint manifestations in type II diabetes mellitus. Ame J Advan Drug Delivery. 3, 59-63 (2015).

Lebiedz-Odrobina D, Kay J. Rheumatic manifestations of diabetes mellitus. Rheum Dis Clin N Am. 36, 681-699 (2010).

Agrawal RP, Gothwal S, Tantia P, et al. Prevalence of rheumatological manifestations in diabetic population from North-West India. J Assoc Physicians India. 62, 788-791 (2014).

Aydeniz A, Gursoy S, Guney E. Which musculoskeletal complications are most frequently seen in type 2 diabetes mellitus? J Inter Med Res. 36, 505-511 (2008).

Kidwai SS, Wahid L, Siddiqi SA, et al. Upper limb musculoskeletal abnormalities in type 2 diabetic patients in low socioeconomic strata in Pakistan. BMC Res Notes. 6, 16 (2013).

Wiwanitkit $S$, Wiwanitkit V. Trigger digits and diabetes mellitus. NAm J Med Sci. 4, 117 119 (2012).

Blom A, Warwick D, Whitehouse M. Apley and Solomon's system of orthopedics and trauma. Taylor and Francis Group. 439440 (2018). 